\title{
NONLINEAR SYSTEM INVERSION APPLIED TO RANDOM VARIABLE GENERATION
}

\author{
A. Pagès-Zamora, M. A. Lagunas, X. Mestre \\ Dpt. Teoria del Senyal i Comunicaciones, Universitat Politècnica de Catalunya (UPC), \\ C/ Gran Capità s/n, Campus Nord UPC, D-5, 08034 Barcelona, Spain \\ alba@gps.tsc.upc.es
}

\begin{abstract}
In this paper, a method to design random variables (rv) generators with the same probability density function (pdf) as a given rv record is presented. The resulting rv generator is a nonlinear system that, when driven by a uniformly distributed $\mathrm{rv}$, provides an output rv with the desired pdf distribution. The analytical description of the desired pdf is not needed; in fact, only a data record of the desired $\mathrm{rv}$ is used Inversion of nonlinear systems and nonlinear system adaptive design are used in this work.
\end{abstract}

\section{INTRODUCTION}

For simulation purposes, generators of random variables (rv) with a given probability distribution function (pdf) are often needed. For instance, in the model for a time-space radio channel of [1], it is shown that the wave azimuth distribution almost matches a Gaussian pdf, whereas their delay distribution approximately fits an exponential pdf. Nevertheless, the measured pdf could not always properly fit an analytic pdf distribution with an acceptable confidence level and over the entire range. For instance, in this radio channel model, the tails of the measured azimuth distribution are not well fit by a normal pdf.

In this paper, a method to design rv generators with the same pdf distribution as a given rv record is presented. As shown below, the resulting rv generator is a nonlinear system (NLS) that, when driven by a uniformly distributed $\mathrm{rv}$, provides an output $\mathrm{rv}$ with the desired pdf distribution. It is important to point out that the analytical description of the desired pdf is not needed; in fact, only a data record of the desired $\mathrm{rv}$ is used. As will be shown, NLS inversion and NLS adaptive design are involved in the design.

The paper is organized as follows. In section II, the "whitening" of a rv is presented. That is, we describe a procedure to obtain a uniformly distributed rv from a given rv record with another pdf. In section III, the rV generator design problem leads to an NLS inversion problem, which is solved adaptatively. Section IV presents simulations and, the paper ends with conclusions in section $\mathrm{V}$.

\section{PDF WHITENING}

In [2], a parallelism between the role that a pdf function plays in nonlinear signal processing and the role that the power spectrum density function plays in linear signal processing is presented.
This relationship allows us to solve the problem of pdf whitening (that is, to obtain a uniformly distributed $\mathrm{rv}$ from another $\mathrm{rv}$ ) similarly to whitening the power spectral density of a stochastic process.

The pdf whitening problem involves the design of an NLS system, denoted by $g[$.$] , that provides a uniformly distributed \mathrm{rv}$ output, denoted hereafter by $u(n)$ with $n$ discrete time index, whenever it is driven by a data record $x(n)$ of a given distribution. Thus, we have,

$$
u(n)=g[x(n)] \text {. }
$$

It is well-known [3] that such a system is,

$$
u(n)=g[x(n)]=2 U_{0}\left[F_{X}(x(n))-1 / 2\right]
$$

with $F_{X}(x)$ the input distribution function and $U_{0}$ the output range, i.e. $u \in\left[-U_{0}, U_{0}\right]$. As (2) is monotonically increasing, the relation between the input pdf, $p_{X}(x)$, and the output one, $p_{U}(u)$, is

$$
p_{U}(u)=p_{X}(x) /(d g(x) / d x)
$$

and it can be stated in the following integral form:

$$
\int_{-\infty}^{\mu} p_{U}(\alpha) \cdot d \alpha=\int_{-\infty}^{x} p_{X}(\lambda) \cdot d \lambda
$$

Assuming that the input range is finite ${ }^{1}$, i.e. $x \in\left[-X_{0}, X_{0}\right]$, and stating the input pdf function $p_{X}(x)$ in terms of the Fourier series approach, expression (4) leads to

$$
\frac{u+U_{0}}{2 U_{0}}=\frac{1}{2 X_{0}} \sum_{k=-\infty}^{+\infty} \Psi_{X}\left(j k \frac{\pi}{X_{0}}\right) \cdot \int_{-X_{0}}^{x} e^{-j k-\frac{\pi}{X_{0}} \lambda} d \lambda
$$

with $\psi_{X}[j v]$ the characteristic function of the rv $x$. Due to the Fourier series periodicity, (5) is valid only for $u$ and $x$ values within their respective ranges. It is straightforward to see that (5) leads to the following pdf whitening system

\footnotetext{
${ }^{1}$ If it were not finite, a truncation of the input range would bi assumed with a certain overflow probability
} 
$u=g[x]=\frac{U_{0}}{X_{0}}\left[x+\sum_{\substack{k=-\infty \\ k \neq 0}}^{+\infty} \psi_{X}\left(j k \frac{\pi}{X_{0}}\right) \frac{e^{-j k \frac{\pi}{X_{0}} x}-(-1)^{k}}{-j k \frac{\pi}{X_{0}}}\right]$.

For practical purposes, the infinite summation in (6) is truncated to $|k| \leq K$ and the characteristic function can be estimated by the sample estimator. Assuming $N$ samples of $x$, the characteristic function estimate could be,

$$
\hat{\psi}_{X}\left(j k \frac{\pi}{X_{0}}\right)=\frac{1}{N} \cdot \sum_{n=1}^{N} e^{j k \frac{\pi}{X_{0}} x(n)}
$$

leading to the approximate pdf whitening system, $\hat{g}[\cdot]$.

$\hat{g}[x(n)]=\frac{U_{0}}{X_{0}}\left[x+\sum_{\substack{k=-K \\ k \neq 0}}^{+K} \hat{\psi}_{X}\left(j k \frac{\pi}{X_{0}}\right) \frac{e^{-j k \frac{\pi}{X_{0}} x(n)}-(-1)^{k}}{-j k \frac{\pi}{X_{0}}}\right]$

Important to remark is that, unlike a simpler whitening system consisting, for instance, of the direct estimation of (2), this pdf whitening system allows a recursive computation of $\hat{\psi}_{X}\left(j k \pi / X_{0}\right)$ and enables the system to whitening nonstationary rv. Additionally, although outside the scope of this paper, it is worth to point out that the previous pdf whitening system can be generalized to an arbitrary number of rv's (see [2] for details).

In order to show the performance of the presented pdf whitening system, 2000 samples of a normally distributed rv $x: \mathrm{N}(0,1)$ are considered. Let us assume that $|x| \leq X_{0}=3$, i.e. an overflow probability of $10^{-3}$ is allowed. From the estimated values of the characteristic function $\hat{\psi}_{X}\left(j k \pi / X_{0}\right)$ for $|k| \leq K=10(7)$ and considering $U_{0}=5$, the pdf whitening system (8) is obtained. Figure 1 shows the normalized histogram of an $x$ data record of 8000 samples and the resulting "whitened" $u$ samples obtained from (8). As seen, the output rv histogram has a flat shape.
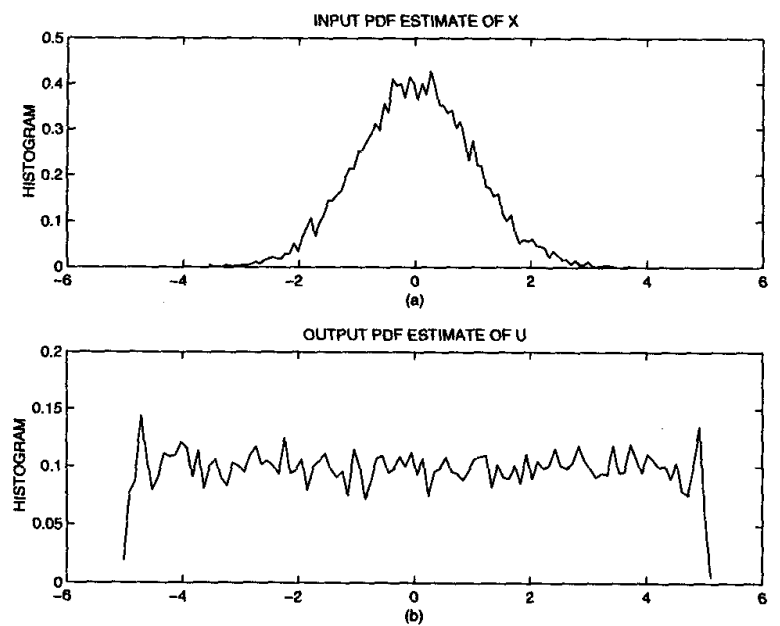

Figure 1. Histograms (8000 samples) of $x$ (a) and $u$ (b).

\section{RV GENERATOR}

From here on, we focus on the problem of designing a NLS system whose output has a given pdf function when it is driven by a uniformly distributed rv. Hereafter, such a system will be referred to as an rv generator.

In the previous section, we showed that a data record of a rv $x$ with a given pdf provides us with a NLS system able to generate a uniformly distributed rv when that NLS system is driven by $x$. Consequently, as shown in Figure 2 , the design of a $x$ rv generator system becomes a nonlinear inverse system design problem of the pdf whitening NLS in (8).

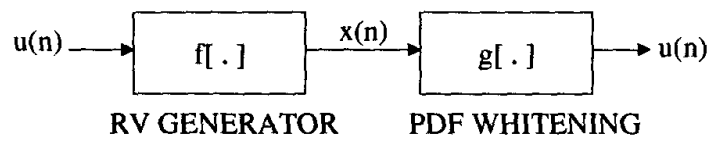

Figure 2. NLS inversion to design the rv generator.

According to (2), the ideal rv generator function $f[u]$ is

$$
f[u]=g^{-1}[u]=F_{X}\left(\frac{u+U_{0}}{2 U_{0}}\right) .
$$

For the sake of comparison, two different NLS designs are considered to model the rv generator: a Volterra model (10) denoted by $f_{V}(u)$ and a trigonometric or Fourier model (11) denoted by $f_{F}(u)[2]$.

$$
\begin{aligned}
& \hat{f}_{V}(u(n))=\sum_{q=0}^{Q} a_{V}(q) \cdot u^{q}(n) \equiv a_{V}^{\prime} \cdot z_{V}(n) \\
& \hat{f}_{F}(u(n))=a(0)+\sum_{q=1}^{Q}\left[a_{F}(2 q) \cdot \cos \left(2 q \omega_{0} u(n)\right)+\right. \\
& \left.+a_{F}(2 q+1) \cdot \sin \left((2 q-1) \omega_{0} u(n)\right)\right] \equiv a_{F}^{\prime} \cdot z_{F}(n)
\end{aligned}
$$

The linearity of both models with respect to the coefficients enables a vector notation as seen in (10) and (11). The vectors of the nonlinear models are $a_{V}$, the Volterra coefficient vector, $\boldsymbol{z}_{\nu}(n)$ the Volterra functional vector consisting of the powers of $u(n), a_{F}$ the Fourier coefficient vector and $z_{F}(n)$ the Fourier functional vector consisting of the sine or cosine functions of $u(n)$. Also in (11) the so-called principal frequency is defined as $\omega_{0}=\pi\left(2 X_{0}\right)$. (See [2] for detail about the Fourier model).

As shown in Figure 3, the design of the rv generator can be accomplished in an adaptive manner by means of the so-called Predistortion-LMS (PLMS) [4]. 


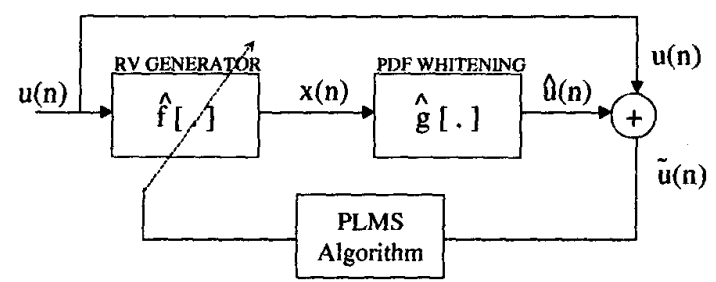

Figure 3. Adaptive design of the rv generator.

The PLMS update of the Volterra or Fourier coefficients follows

$$
a(n+1)=a(n)+\frac{\mu}{p(n)} \cdot \tilde{u}(n) \cdot \nabla_{x} \hat{g}\left(\left.x\right|_{x(n)} \cdot z(n),\right.
$$

substituting $a(\mathrm{n})$ for the respective coefficient vector and $z(n)$ for the respective functional vector, as defined in (10) for the Volterra model and in (11) for the Fourier model. In (12) $\mu$ is the step-size parameter, $\tilde{u}(n)$ is the error signal,

$$
\tilde{u}(n)=u(n)-\hat{u}(n)
$$

and $p(n)$ is the estimate of the power of the functionals,

$$
p(n+1)=\beta \cdot p(n)+(1-\beta) \cdot z^{\prime}(n) \cdot z(n) \text {. }
$$

The PLMS adaptive algorithm is a gradient algorithm useful in NLS inversion problems because it includes, due to the chain rule, the gradient $\nabla_{x} \hat{g}(x)$ of the function to be inverted. From (2), the gradient depends on $p_{X}(x)$.

$$
\nabla_{x} g[x]=2 U_{0} \cdot \nabla_{x} g\left[F_{X}(x)\right]=2 U_{0} \cdot p_{X}(x)
$$

For practical purposes, the gradient of $(8)$ can be used directly. Different pdf estimates could be also taken into account to estimate de gradient [2].

The design of the rv generator could have also been performed in reverse order, that is, $\hat{g}(\cdot)$ could have been put in front of the $\hat{f}(\cdot)$ in Figure 3 . In that case, a least square solution of the NLS model of the rv generator would be feasible because the signal error is linear with the coefficients. The limitation is that, in the reverse order, a large record of $x$ would be needed, and the objective of the paper is precisely to design a generator of the $x$ rv from a small record of $x$.

\section{SIMULATIONS}

Two sets of simulations are included. The first one uses the actual characteristic function, whereas in the second simulation only a record of a $x \mathrm{rv}$ is assumed.

First, let us consider a Laplacian rv whose pdf is,

$$
p_{X}(x)=\alpha / 2 \cdot e^{-\alpha|x|}
$$

with parameter $\alpha$ set to 1 . The pdf whitening system is built from expression (8) with $U_{0}=1$ and using the actual samples of the characteristic function for $K=10$.

$$
\psi_{X}\left(j k \frac{\pi}{X_{0}}\right)=\frac{\alpha^{2}}{\alpha^{2}+k \frac{\pi}{X_{0}}} \quad|k|<K=10
$$

Due to symmetry of the distribution function, the pdf whitening system and the inverse system both have odd input/output relations. Thus, the Volterra system (10) that models the rv generator only keeps the odd powers, whereas the Fourier model keeps only the sine functionals. Both models consist of 15 coefficients.

A 5000-length data record of a uniformly distributed rv is used in the adaptive design of the rv generator (3). The PLMS parameters (12) (14) are set to $\mu=2$ and $\beta=0.99$ for both models. The gradient function (15) is computed using the Laplacian pdf function (16). The final relations of $\hat{f}_{V}(u)$ and $\hat{f}_{F}(u)$ together with the ideal ones (dashed line) are shown in figure (4.a) and (4.b).

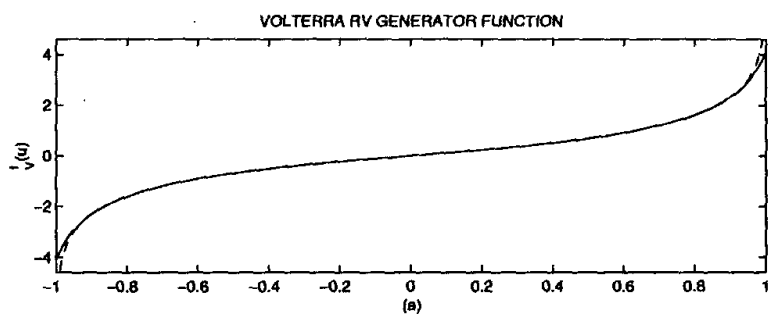

FOURIER RV GENERATOR FUNCTION

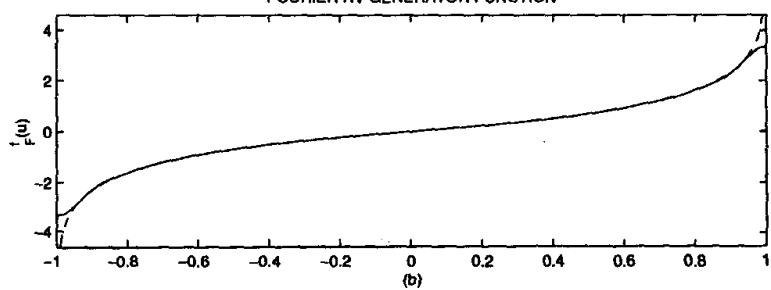

Figure 4. Ideal rv generator in dashed line. In solid line, Volterra (a) and Fourier (b) rv generator functions.

Figure (5) compares the Laplacian pdf (dashed line) to the output rv histogram of the Volterra rv generator (Fig. 5.a) and Fourier rv generator (Fig. 5.b). Both histograms have been computed from $2 \cdot 10^{4}$ length data records.

Although not shown, the convergence of the Fourier coefficients is faster than that of the Volterra coefficients, but the Fourier model does not properly fit the tails of the Laplacian pdf function (Fig. 5.b). This is due to the fact that the ideal function $f[u]$ has a sharp behavior at the boundaries of the input range (see Fig. 4) that the Fourier model does not match properly. In this case, the Volterra model provides better performance for such a NLS design. 

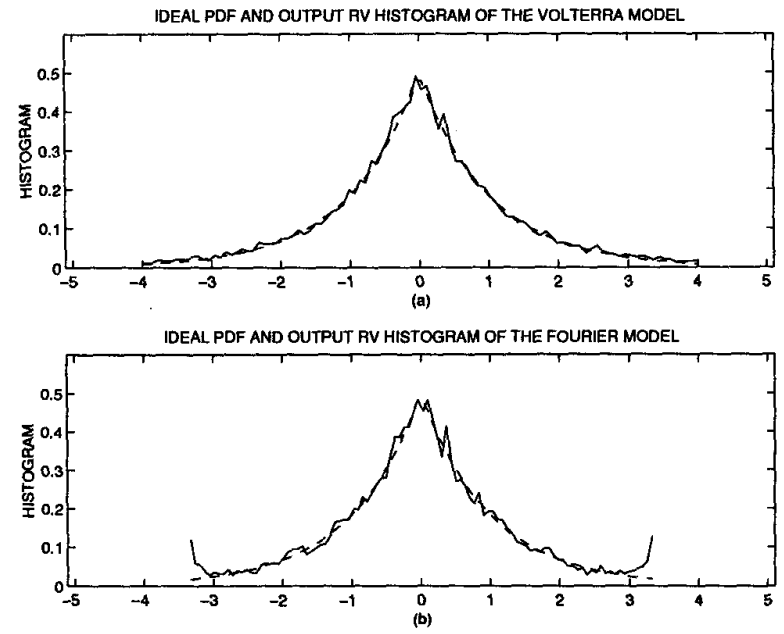

Figure 5. Laplacian pdf function (dashed line). In the solid line, the output histogram of the Volterra (a) and Fourier (b) rv generator systems.

The second set of simulations uses only 2000 samples of a normal distributed rv $x: \mathrm{N}(0,1)$. As shown in section 2 , this data record allows the design of the pdf whitening system (in this case $U_{0}=1$ ). Once the pdf whitening system is obtained, the rv generator can be adaptatively designed using the scheme of Figure 3.

For that purpose, a Volterra system $(10), \hat{f}_{V}(u)$, with $Q=15$ is considered to model the rv generator. The coefficients are updated with $2 \cdot 10^{4}$ samples of a uniformly distributed rv $u$ and by means of the PLMS adaptive algorithm with $\mu=2$ and $\beta=0.99$. The Fourier series approximation of $p_{X}(x)(K=10)$ is used to compute gradient function, $\nabla_{x} \hat{g}(x)$.
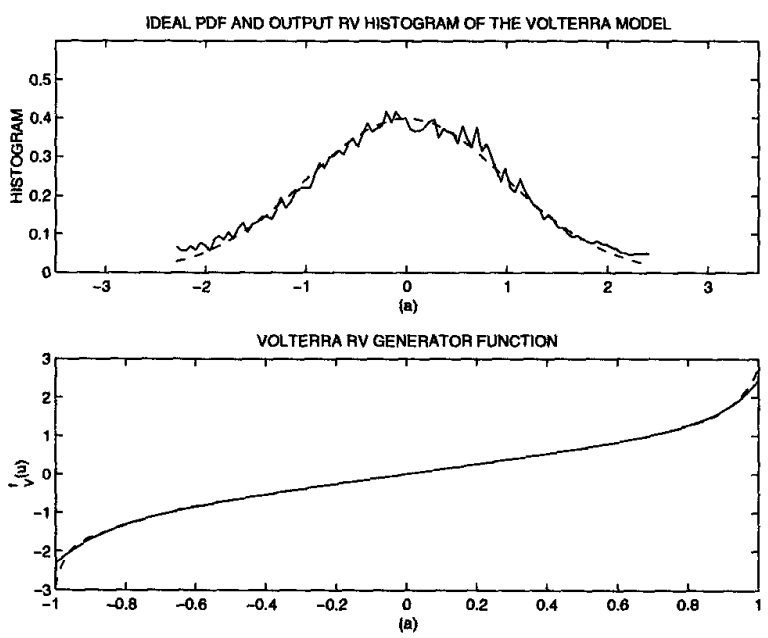

Figure 6. (a) Ideal pdf (dashed line) and histogram of the rv generator output (solid line). (b) Ideal rv generator function (dashed line) and actual rv generator function (solid line).
Figure (6.b) shows the ideal input/output relation of the rv generator system in the dashed line along with the final one achieved by the Volterra system after the adaptive design. Additionally, figure (6.a) shows the actual pdf (dashed line) and the histogram of the Volterra rv generator output using $2 \cdot 10^{4}$ samples.

\section{REMARKS}

This paper shows how a nonlinear system that generates a rv with a given pdf can be designed from knowledge only of a data record of such a rv. It has been shown that data records of 2000 samples are large enough to obtain a reliable rv generator system. As a preliminary step, we also presented the design of nonlinear systems that are able to provide a uniformly distributed $\mathrm{rv}$ at the output when driven by an input signal with a given pdf.

\section{ACKNOWLEDGEMENTS}

This work has been supported by CICYT: TIC96-0500-C10-01, TIC98-0412, TIC98-0703 and CIRIT: 1998SGR-00081

\section{REFERENCES}

[1] Pedersen K.I., Mogensen P.E., Fleury B.H., "A Stochastic Model of the Temporal and Azimuthal Dispersion seen at the Base Station in Outdoor Propagation Environments", Submitted to Trans VTC, 1999.

[2] Pagès-Zamora A, Lagunas M.A, "Fourier Models for Non-Linear Signal Processing", EURASIP Signal Processing, Vol. 76, pp.1-16, June 1999.

[3] A. Papoulis, Probability, Random variable sand Stochastic Processes, McGraw-Hill, USA, 1965.

[4] Stonick J.T. et al., "Memoryless Polynomial Adaptive Predistortion", Proc. IEEE-ICASSP95, pp. 981-984, Detroit, Michigan, USA, May 1995. 